\title{
Usefulness of plasminogen activator inhibitor- 1 as a predictive marker of mortality in sepsis
}

Kota Hoshino*, Taisuke Kitamura, Yoshihiko Nakamura, Yuhei Irie, Norihiko Matsumoto, Yasumasa Kawano and Hiroyasu Ishikura

\begin{abstract}
Background: Sepsis is one of the most significant causes of mortality in intensive care units. It indicates crosstalk between inflammation and coagulation. In this study, we aimed to identify prognostic markers among sepsis biomarkers and coagulation/fibrinolysis markers.

Methods: Patients with sepsis according to the Sepsis-3 criteria were enrolled from January 2013 to September 2015. Univariate and multivariate logistic regression analyses were performed to identify an independent predictive marker of 28-day mortality among sepsis biomarkers and coagulation/fibrinolysis markers on ICU admission. Receiver operating characteristic analysis was performed; the optimal cutoff value of 28-day mortality was calculated using the predictive marker. Patients were classified into two groups according to the cutoff level of the predictive marker. Patient characteristics were compared between the groups.
\end{abstract}

Results: A total of 186 patients were enrolled in this study; the 28-day mortality was 19.4\% (36/186). PAl-1 was identified as the only independent predictive marker of 28-day mortality by univariate and multivariate logistic regression. The area under the curve was 0.72 ; the optimal cutoff level was $83 \mathrm{ng} / \mathrm{ml}$ (sensitivity, 75\%; specificity, 61\%). Patients were classified into a higher group (PAl-1 level $\geq 83 \mathrm{ng} / \mathrm{ml} ; n=85$ ) and a lower group (PAl-1 level $<83 \mathrm{ng} / \mathrm{ml} ; n=101$ ). All disseminated intravascular coagulation (DIC) scores and Sequential Organ Failure Assessment score were significantly higher in the higher group than in the lower group.

Conclusions: PAI-1 can predict prognosis in sepsis patients. PAI-1 reflects DIC with suppressed fibrinolysis and organ failure, with microthrombi leading to microcirculatory dysfunction.

Keywords: Disseminated intravascular coagulation, Fibrinolysis, Pathogen-associated molecular patterns, Sepsis-3

\section{Background}

Sepsis is one of the most significant causes of mortality in intensive care units [1], and mortality among septic shock patients has been reported to be $30-50 \%[2,3]$. To improve the prognosis of sepsis patients, it is important to diagnose and immediately treat sepsis. The usefulness of sepsis biomarkers, such as procalcitonin (PCT) and presepsin (PSEP), has been reported; PCT and PSEP have been reported to be superior to $\mathrm{C}$-reactive protein (CRP) and interleukin-6 for sepsis diagnosis and assessment of sepsis severity [4-6].

\footnotetext{
* Correspondence: hoshinoqq@yahoo.co.jp

Department of Emergency and Critical Care Medicine, Faculty of Medicine, Fukuoka University, 7-45-1 Nanakuma, Jonan-ku, Fukuoka 814-0180, Japan
}

The defensive role of thrombosis is referred to as immunothrombosis [7]. Immunothrombosis designates an innate immune response induced by the formation of thrombi in microvessels. However, if left uncontrolled, immunothrombosis can eventually lead to disseminated intravascular coagulation (DIC) $[8,9]$. Previous studies reported that the frequency of DIC in sepsis patients was $20-40 \%$ [10-13]. Therefore, it is important to measure coagulation/fibrinolysis markers as well as sepsis biomarkers in sepsis to assess the presence of crosstalk between inflammation and coagulation.

In this study, we aimed to identify prognostic markers among sepsis biomarkers and coagulation/fibrinolysis markers. 


\section{Methods}

\section{Patient selection}

This retrospective single-center study was approved by the ethics committee of Fukuoka University Hospital (No. 16-3-14). The criteria for admission to the ICU in patients with sepsis include one or more organ failures including shock or disturbance of consciousness. Patients with sepsis were enrolled from January 2013 to September 2015. The diagnosis of sepsis was based on the definition of Sepsis-3 [14]. The exclusion criteria were age $<18$ years, presence of leukemia, liver cirrhosis, and cardiopulmonary arrest on admission and occurrence of death within $24 \mathrm{~h}$ of ICU admission. Patients were classified into non-survivor and survivor groups on day 28 of ICU admission (Fig. 1).

\section{Patient characteristics}

The two groups of patients were compared in terms of age, sex, infection focus, vital signs, the Japanese Association for Acute Medicine DIC score [15] along with the positive rate, the Sequential Organ Failure Assessment (SOFA) score [16] on ICU admission, and therapeutic agents. Moreover, sepsis biomarkers and coagulation/fibrinolysis markers were compared between non-survivor and survivor groups.

\section{Identification of predictive markers}

In this study, we examined sepsis biomarkers and coagulation/fibrinolysis markers in blood samples on ICU admission. First, univariate logistic regression analyses were performed. The explanatory variables were CRP, PCT, and PSEP as sepsis biomarkers and platelet counts, prothrombin time international normalized ratio (PTINR), activated partial thromboplastin time, antithrombin, D-dimer, thrombin-antithrombin complex (TAT), plasmin- $\alpha 2$ plasmin inhibitor complex, protein $C(\mathrm{PC})$, thrombomodulin (TM), soluble fibrin (SF), and plasminogen activator inhibitor-1 (PAI-1) as coagulation/fibrinolysis markers. The response variable was 28-day mortality. Subsequently, multivariate logistic regression

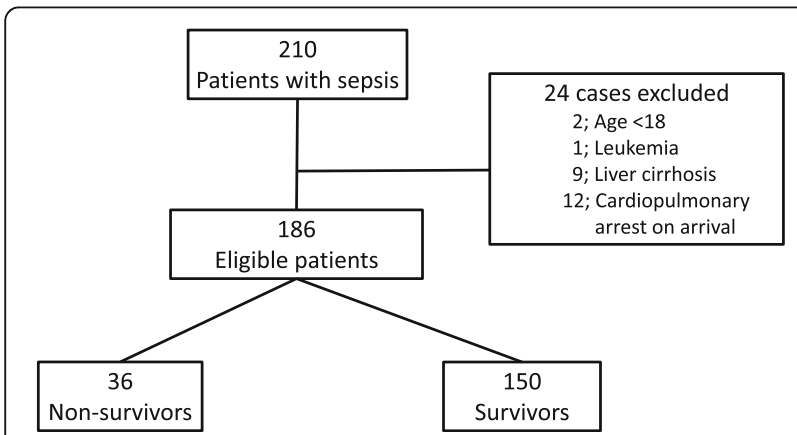

Fig. 1 Flow chart. Flow diagram of patients who met the inclusion/ exclusion criteria for the study population analysis was performed to identify the independent predictive marker of 28-day mortality using the markers that were identified as significant in univariate logistic regression.

\section{Cutoff value of the predictive marker and relationship with each score}

Receiver operating characteristic (ROC) analysis was performed and the optimal cutoff value of 28-day mortality was calculated using the marker that was selected in multivariate logistic regression.

\section{Relationship between the predictive marker and sepsis severity}

We divided patients into the following two groups considering the optimal cutoff value: the higher group and the lower group. Patient characteristics were compared between these two groups. The correlations of the predictive marker with DIC and SOFA scores were examined to evaluate the relationship between the predictive marker and each score. In addition, the 28-day survival rate was compared between the higher and the lower groups using the Kaplan-Meier analysis.

\section{Time course of sepsis biomarkers and coagulation/ fibrinolysis markers}

Time course of sepsis biomarkers and coagulation/fibrinolysis markers that were significantly different according to the univariate analyses were compared between the non-survivor and survivor groups.

\section{Assay of sepsis biomarkers and coagulation/fibrinolysis markers}

Blood samples were routinely collected for measuring markers, and there were no lack of data on ICU admission in this study. CRP levels were measured by CRPLATEX (II) X2 "SEIKEN" (Denka Seiken Co., Ltd, Tokyo, Japan) using EDTA plasma as a sample. PCT levels were measured by the Elecsys BRAHMS PCT assay (Roche Diagnostics, Tokyo, Japan) using EDTA plasma as a sample. PSEP levels were measured using a compact-automated immunoanalyzer, PATHFAST, based on a chemiluminescent enzyme immunoassay (CLEIA) (Mitsubishi Chemical Medience Corp., Japan). Platelet counts were measured in whole blood using an XT1800i (Sysmex Co., Kobe, Japan). PT, APTT, AT, Ddimer, PIC, PC, and SF levels were measured in the plasma using a Coapresta 2000 (Sekisui Medicak, Tokyo, Japan). TAT, TM, and PAI-1 levels were measured using a STACIA (Mitsubishi Chemical Medience Corp., Tokyo, Japan). Total PAI-1 including active PAI-1 and tPA-PAI1 complex was defined as PAI-1 in this study. 


\section{Statistical analysis}

Continuous variables are presented as median (interquartile range). Comparisons between groups were performed using the chi-square test for dichotomous variables and Mann-Whitney $U$ test for continuous variables. ROC analysis, including determination of the area under the curve (AUC), was performed to determine the significance of the marker level for predicting 28-day mortality. The Youden index was used to identify the cutoff value. Correlations between the predictive marker and each score were evaluated using Spearman's rank test. A $P$ value of $<0.05$ was considered statistically significant. All statistical analyses were performed using JMP version 12 (SAS institute Japan, Tokyo, Japan).

\section{Results}

\section{Patient selection}

Patient enrollment into the study and exclusion from the study are shown in Fig. 1. Twenty-four of the 210 patients were excluded according to exclusion criteria. A total of 186 patients were enrolled in this study, and the 28 -day mortality rate was $19.4 \%(36 / 186)$. Of the 186 patients, 36 patients were classified in the non-survivor group and 150 in the survivor group.

\section{Patient characteristics}

Patient characteristics are presented in Table 1. There were significant differences in age, infection focus, SOFA score (in particular, cardiovascular and renal SOFA scores), and continuous renal replacement therapy; however, there was no significant difference among other patient characteristics. With respect to the comparison of sepsis biomarkers and coagulation/fibrinolysis markers, PCT, PT-INR, APTT, TAT, SF, and PAI-1 levels were significantly higher in the non-survivor group than in the survivor group. On the other hand, AT and PC levels were significantly lower in the non-survivor group than in the survivor group (Table 2).

\section{Identification of predictive markers}

PSEP $(P<0.05)$ as a sepsis biomarker, TAT $(P<0.05)$, PC $(P<0.01)$, SF $(P<0.01)$, and PAI- $(P<0.01)$ as coagulation/fibrinolysis markers, and SOFA score $(P<0.01)$ were selected in univariate logistic regression (Table 3 ). Subsequently, multivariate logistic regression was performed using PSEP, TAT, PC, SF, PAI-1, and SOFA score as explanatory variables. PAI- 1 was found to be the only independent predictive marker of 28 -day mortality $(P<$ 0.05; Table 3).

\section{Cutoff value of the predictive marker and relationship with each score}

With regard to the accuracy of predicting 28-day mortality based on the level of PAI-1 in the ROC analysis, the AUC was 0.72 and the optimal cutoff value was $83 \mathrm{ng} / \mathrm{ml}$ (sensitivity, 75\%; specificity, 61\%).

\section{Relationship between the predictive marker and sepsis severity}

Patients were classified into the higher group (PAI-1 level $\geq 83 \mathrm{ng} / \mathrm{ml} ; n=85$ ) and the lower group (PAI-1 level $<83 \mathrm{ng} / \mathrm{ml} ; n=101$ ). DIC, SOFA scores (in particular, cardiovascular and coagulation SOFA scores), and 28day mortality were significantly higher in the higher group than those in the lower group (all $P<0.01$; Table 4). To determine which scores affect the PAI-1 level the most, correlations between the PAI-1 level and DIC or SOFA score were evaluated using Spearman's rank test. We noted positive correlations between the PAI-1 level and DIC score $(r=0.18, P<0.05)$ or SOFA score $(r=0.32, P<0.01)$, in particular, cardiovascular $(r=0.35, \quad P<0.01)$ and renal $(r=0.22, \quad P<0.01$; Table 5). The 28-day survival rate according to Kaplan-Meier analysis was significantly lower in the higher group than that in the lower group (log-rank test, $P<0.01$; Fig. 2).

\section{Time course of sepsis biomarkers and coagulation/ fibrinolysis markers}

Figure 3 shows the time courses of PSEP, TAT, PC SF, and PAI-1 that were significantly different according to univariate analyses. PC and PAI-1 levels were significantly different between the non-survivor and survivor groups on days 0,3 , and $7(P<0.05)$. In particular, PAI- 1 levels at all time courses were significantly higher in the non-survivor group than those in the survivor group $(P<0.01)$.

\section{Discussion}

In this study, we used the new sepsis definition "Sepsis3 " and identified the predictive marker of 28-day mortality among sepsis biomarkers and coagulation/fibrinolysis markers. This study revealed that PAI-1 was the most predictive marker according to the multivariate analysis, and the cutoff value was $83 \mathrm{ng} / \mathrm{ml}$. Morbidities of DIC and organ dysfunctions were significantly higher in the higher PAI-1 group. Moreover, PAI-1 was correlated with SOFA score, in particular cardiovascular and renal scores. PAI-1 levels were significantly higher in the nonsurvivor group than those in the survivor group during the time course as well as on admission.

The first definition of septic syndrome established in 1992 was based on the concomitant presence of presumed/confirmed infection and at least two of the four Systemic Inflammatory Response Syndrome criteria [17]. The new sepsis definition "Sepsis-3" mentions lifethreatening organ dysfunction caused by a dysregulated host response to infection [14]. Therefore, it is important to assess organ dysfunction that is caused by 
Table 1 Comparison of patient characteristics

\begin{tabular}{|c|c|c|c|c|}
\hline & $\begin{array}{l}\text { Total } \\
n=186\end{array}$ & $\begin{array}{l}\text { Non-survivors } \\
n=36\end{array}$ & $\begin{array}{l}\text { Survivors } \\
n=150\end{array}$ & $P$ value \\
\hline Age (years old) & $72(62-79)$ & $76(68-81)$ & $71(61-78)$ & $<0.05$ \\
\hline Male & $115(62)$ & $20(56)$ & $95(63)$ & 0.39 \\
\hline Infection focus & & & & $<0.05$ \\
\hline Lung & $77(41)$ & $8(22)$ & $69(46)$ & \\
\hline Abdomen & $61(33)$ & $21(58)$ & $40(27)$ & \\
\hline Skin and soft tissue & $13(7)$ & $3(8)$ & $10(7)$ & \\
\hline Urinary tract & $11(6)$ & $2(6)$ & $9(6)$ & \\
\hline Others & $11(6)$ & $1(3)$ & $10(7)$ & \\
\hline Unknown & $13(7)$ & $1(3)$ & $12(8)$ & \\
\hline Mean blood pressure $(\mathrm{mmHg})$ & $78(67-97)$ & $74(63-92)$ & $79(67-97)$ & 0.13 \\
\hline Heart rate (bpm) & $110(95-120)$ & $111(95-120)$ & $110(95-121)$ & 0.77 \\
\hline Respiratory rate (bpm) & $23(19-28)$ & $24(20-28)$ & $22(18-28)$ & 0.28 \\
\hline Body temperature $\left({ }^{\circ} \mathrm{C}\right)$ & $36.9(36.3-37.9)$ & $36.7(36.0-37.4)$ & $36.9(36.4-38.1)$ & 0.06 \\
\hline JAAM DIC score & $3(2-5)$ & $4(2-6)$ & $3(2-5)$ & 0.13 \\
\hline JAAM DIC positive rate & $87(47)$ & $21(58)$ & $66(44)$ & 0.12 \\
\hline SOFA score & $8(5-11)$ & $11(8-13)$ & $8(5-11)$ & $<0.01$ \\
\hline Respiratory & $2(2-3)$ & $2(1-3)$ & $2(2-3)$ & 0.87 \\
\hline Cardiovascular & $1(0-4)$ & $4(0-4)$ & $1(0-4)$ & $<0.01$ \\
\hline Liver & $0(0-1)$ & $0(0-1)$ & $0(0-1)$ & 0.95 \\
\hline Renal & $1(0-3)$ & $3(1-4)$ & $1(0-3)$ & $<0.01$ \\
\hline Coagulation & $1(0-2)$ & $0(0-2)$ & $1(0-2)$ & 0.85 \\
\hline CNS & $1(0-2)$ & $1(0-3)$ & $1(0-2)$ & 0.95 \\
\hline rhs TM & $46(25)$ & $11(31)$ & $35(23)$ & 0.37 \\
\hline AT III & $28(15)$ & $6(17)$ & $22(15)$ & 0.76 \\
\hline IVIG & $92(49)$ & $21(58)$ & $71(47)$ & 0.24 \\
\hline CRRT & $44(24)$ & $17(47)$ & $27(18)$ & $<0.01$ \\
\hline PMX-DHP & $28(15)$ & $9(25)$ & 19 (13) & 0.06 \\
\hline
\end{tabular}

Data are presented as median (interquartile range) or number (percentage)

JAAM Japanese Association for Acute Medicine, DIC disseminated intravascular coagulation, SOFA sequential organ failure assessment, CNS central nervous system, rhs TM recombinant human soluble thrombomodulin, $A T$ antithrombin, IVIG intravenous immunoglobulin, CRRT continuous renal replacement therapy, PMX-DHP direct hemoperfusion with polymyxin B-immobilized fiber

coagulo-fibrinolytic abnormalities in sepsis. This is the first study to evaluate the prognostic value of sepsis biomarkers and coagulation/fibrinolysis markers using the Sepsis-3 definition.

There are some reports that sepsis biomarkers are useful for predicting mortality in patients with sepsis. Clec'h et al. [18] reported that PCT was able to predict mortality in patients with septic shock. Jensen et al. [19] reported that PCT level increase denoted a high risk of mortality. In the multicenter, retrospective, case-control study, PSEP was the only predictive marker of mortality according to multivariate analysis including PCT and PSEP [20]. In this study, PSEP was significant according to the univariate analysis; however, PSEP was not the predictive marker according to the multivariate analysis.
In sepsis, innate immune responses start following the identification of pathogen-associated molecular patterns or damage-associated molecular patterns by patternrecognition receptors, such as Toll-like receptors expressed on immunocompetent cells and the endothelium. The sensed danger signals activate both intracellular signal transduction pathways and plasma cascades, which together produce pro-inflammatory cytokines, further stimulating the production of inflammatory biomarkers. The actions of inflammatory cytokines trigger the production of large amounts of tissue factor from monocytes/macrophages and the vascular endothelium, thus leading to marked coagulation activation. PAI-1 is synthesized by endothelial cells and hepatocytes. It is the main inhibitor of tissue-type plasminogen activator and 
Table 2 Comparison of sepsis biomarkers and coagulation/fibrinolysis markers

\begin{tabular}{|c|c|c|c|c|}
\hline & $\begin{array}{l}\text { Total } \\
n=186\end{array}$ & $\begin{array}{l}\text { Non-survivors } \\
n=36\end{array}$ & $\begin{array}{l}\text { Survivors } \\
n=150\end{array}$ & $P$ value \\
\hline $\mathrm{CRP}(\mathrm{mg} / \mathrm{dl})$ & $12(5-20)$ & $15(9-21)$ & $12(4-20)$ & 0.21 \\
\hline PCT (ng/ml) & $7(1-42)$ & $18(2-89)$ & $6(1-33)$ & $<0.05$ \\
\hline PSEP (pg/ml) & $839(440-1597)$ & $905(546-1811)$ & $815(422-1562)$ & 0.12 \\
\hline Platelet count $(\times 104 / \mu l)$ & $14.8(8.6-23.0)$ & $16.6(5.8-25.3)$ & $14.4(9.0-22.3)$ & 0.81 \\
\hline PT-INR & $1.3(1.2-1.6)$ & $1.5(1.3-2.1)$ & $1.3(1.2-1.5)$ & $<0.05$ \\
\hline APTT (s) & $35(30-42)$ & $39(33-45)$ & $34(30-41)$ & $<0.05$ \\
\hline AT (\%) & $70(56-87)$ & $63(48-77)$ & $73(58-87)$ & $<0.05$ \\
\hline D-dimer $(\mu \mathrm{g} / \mathrm{ml})$ & $6.8(2.9-15.7)$ & $8.4(3.3-18.2)$ & $6.7(2.8-14.6)$ & 0.27 \\
\hline TAT (ng/ml) & $6.9(3.7-16.5)$ & $8.9(5.5-34.8)$ & $6.5(3.2-13.0)$ & $<0.05$ \\
\hline $\mathrm{PIC}(\mu \mathrm{g} / \mathrm{ml})$ & $1.7(1.0-3.3)$ & $1.5(0.8-3.2)$ & $1.7(1.1-3.4)$ & 0.19 \\
\hline PC (\%) & $47(33-70)$ & $38(26-56)$ & $52(34-70)$ & $<0.01$ \\
\hline $\mathrm{TM}(\mathrm{U} / \mathrm{ml})$ & $33(23-47)$ & $40(25-52)$ & $32(23-45)$ & 0.12 \\
\hline $\mathrm{SF}(\mu \mathrm{g} / \mathrm{ml})$ & $21(12-62)$ & $45(17-80)$ & $20(12-43)$ & $<0.01$ \\
\hline PAl-1 (ng/ml) & $66(29-191)$ & $154(73-527)$ & $51(26-154)$ & $<0.01$ \\
\hline
\end{tabular}

Data are presented as median (interquartile range)

CRP C-reactive protein, PCT procalcitonin, PSEP presepsin, PT-INR prothrombin time-international normalized ratio, APTT activated partial thromboplastin time, AT antithrombin, TAT thrombin-antithrombin complex, PIC plasmin a2-plasmin inhibitor complex, $P C$ protein $C, T M$ thrombomodulin, SF soluble fibrin, $P A I-1$ plasminogen activator inhibitor-1

plays an important role in the regulation of fibrinolysis. Elevated levels of PAI-1 result in deficient plasminogen activation and are a risk factor for thrombosis, including DIC. It is already known that PAI-1 level is markedly increased in sepsis, fibrinolysis is strongly suppressed, and dissolution of multiple microthrombi is more difficult [21], and because of microcirculatory impairment, severe organ dysfunction may occur [22, 23]. On the other hand, PAI-1 is not involved in malignant tumors such as acute leukemia and solid cancers [23].

Koyama et al. [24] reported that TAT, PC, and PAI-1 were predictive markers of mortality in patients with sepsis. TAT, PC, and PAI-1 were significant according to the univariate analysis as well as a previous study [24],

Table 3 Logistic regression analyses of the coagulation/fibrinolysis markers for 28-day mortality

\begin{tabular}{|c|c|c|c|c|c|c|}
\hline \multirow[b]{2}{*}{ Markers } & \multicolumn{3}{|c|}{ Univariate analyses } & \multicolumn{3}{|c|}{ Multivariate analyses } \\
\hline & $\mathrm{OR}$ & $95 \% \mathrm{Cl}$ & $P$ value & $\mathrm{OR}$ & $95 \% \mathrm{Cl}$ & $P$ value \\
\hline CRP (mg/dl) & 1.020 & $0.986-1.054$ & NS & & & \\
\hline PCT (ng/ml) & 1.000 & $0.993-1.003$ & NS & & & \\
\hline PSEP (pg/ml) & 1.000 & $1.000-1.000$ & $<0.05$ & 1.000 & $0.999-1.000$ & NS \\
\hline Platelet count $\left(\times 10^{4} / \mu l\right)$ & 1.003 & $0.973-1.028$ & NS & & & \\
\hline PT-INR & 0.993 & $0.834-1.034$ & NS & & & \\
\hline APTT (s) & 1.013 & $0.992-1.033$ & NS & & & \\
\hline AT (\%) & 0.983 & $0.966-1.000$ & NS & & & \\
\hline D-dimer $(\mu \mathrm{g} / \mathrm{ml})$ & 1.005 & $0.995-1.015$ & NS & & & \\
\hline TAT (ng/ml) & 1.015 & $1.003-1.027$ & $<0.05$ & 0.999 & $0.982-1.014$ & NS \\
\hline $\mathrm{PIC}(\mu \mathrm{g} / \mathrm{ml})$ & 1.021 & $0.940-1.094$ & NS & & & \\
\hline PC (\%) & 0.977 & $0.959-0.993$ & $<0.01$ & 0.986 & $0.967-1.004$ & NS \\
\hline $\mathrm{TM}(\mathrm{U} / \mathrm{ml})$ & 1.005 & $0.996-1.014$ & NS & & & \\
\hline $\mathrm{SF}(\mu \mathrm{g} / \mathrm{ml})$ & 1.021 & $1.008-1.034$ & $<0.01$ & 1.014 & $0.997-1.031$ & NS \\
\hline PAl-1 (ng/ml) & 1.002 & $1.001-1.003$ & $<0.01$ & 1.002 & $1.000-1.003$ & $<0.05$ \\
\hline SOFA score & 1.200 & $1.078-1.348$ & $<0.01$ & 1.075 & $0.943-1.231$ & NS \\
\hline
\end{tabular}

$O R$ odds ratio, $C l$ confidence interval, $C R P$ C-reactive protein, $P C T$ procalcitonin, $P S E P$ presepsin, $P T$-INR prothrombin time-international normalized ratio, APTT activated partial thromboplastin time, $A T$ antithrombin, $T A T$ thrombin-antithrombin complex, PIC plasmin a2-plasmin inhibitor complex, $P C$ protein $C_{i}$ TM thrombomodulin, SF soluble fibrin, PAI-1 plasminogen activator inhibitor-1, SOFA sequential organ failure assessment 
Table 4 Patient characteristics of the PAl-1 higher and lower groups

\begin{tabular}{llll}
\hline & $\begin{array}{l}\text { PAl-1 } \geq 83 \mathrm{ng} / \mathrm{ml} \\
n=85\end{array}$ & $\begin{array}{l}\text { PAl-1 }<83 \mathrm{ng} / \mathrm{ml} \\
n=101\end{array}$ & $\begin{array}{l}P \text { value } \\
\text { Age }\end{array}$ \\
Male & $50(53-80)$ & $71(61-78)$ & 0.14 \\
JAAM DIC score & $4(2-6)$ & $65(64)$ & 0.44 \\
JAAM DIC positive rate & $52(61)$ & $2(1-5)$ & $<0.01$ \\
SOFA score & $10(8-12)$ & $75(35)$ & $<0.01$ \\
Respiratory & $2(2-3)$ & $2(1-3)$ & $<0.01$ \\
Cardiovascular & $4(0-4)$ & $0(0-3)$ & 0.42 \\
Liver & $0(0-1)$ & $0(0-0)$ & $<0.01$ \\
Renal & $2(0-3)$ & $1(0-3)$ & 0.09 \\
Coagulation & $1(0-2)$ & $0(0-1)$ & 0.18 \\
CNS & $1(1-3)$ & $1(0-2)$ & 0.01 \\
28-day mortality & $27(32)$ & $9(9)$ & $<0.01$ \\
\hline
\end{tabular}

Data are presented as median (interquartile range) or number (percentage) PAI-1 plasminogen activator inhibitor-1, JAAM Japanese Association for Acute Medicine, DIC disseminated intravascular coagulation, SOFA sequential organ failure assessment, CNS central nervous system

and PAI-1 was finally the only predictive marker in this study. Madoiwa et al. [25] examined 117 patients with sepsis-induced DIC and reported that the hazard ratio of 28-day mortality was increasing 23 times with a PAI-1 level of $>90 \mathrm{ng} / \mathrm{ml}$. The $90 \mathrm{ng} / \mathrm{ml}$ PAI-1 level is similar to the PAI-1 cutoff value of $83 \mathrm{ng} / \mathrm{ml}$ in this study. A previous study [24] revealed that the PAI-1 cutoff value was $269 \mathrm{ng} / \mathrm{ml}$; however, the PAI-1 cutoff value in this study was $83 \mathrm{ng} / \mathrm{ml}$. There was a huge difference in the PAI-1 cutoff value between the two studies. Although there is no unified method for PAI-1 level measurement, there are a few reagents for this measurement. The difference in PAI-1 cutoff values may be affected by reagents.

When considering the PAI-1 cutoff level, DIC and SOFA scores were significantly higher in patients with a PAI-1 level of $\geq 83 \mathrm{ng} / \mathrm{ml}$ than in those with a PAI-1 level

Table 5 Correlations between PAI-1 and each score

\begin{tabular}{lcl}
\hline & Correlation coefficient & $P$ value \\
\hline JAAM DIC score & 0.18 & $<0.05$ \\
SOFA score & 0.32 & $<0.01$ \\
Respiratory & -0.01 & 0.90 \\
Cardiovascular & 0.35 & $<0.01$ \\
Liver & 0.06 & 0.40 \\
Renal & 0.22 & $<0.01$ \\
Coagulation & 0.13 & 0.08 \\
CNS & 0.13 & 0.08 \\
\hline
\end{tabular}

PAl-1 plasminogen activator inhibitor-1, JAAM Japanese Association for Acute Medicine, DIC disseminated intravascular coagulation, SOFA sequential organ failure assessment, CNS central nervous system

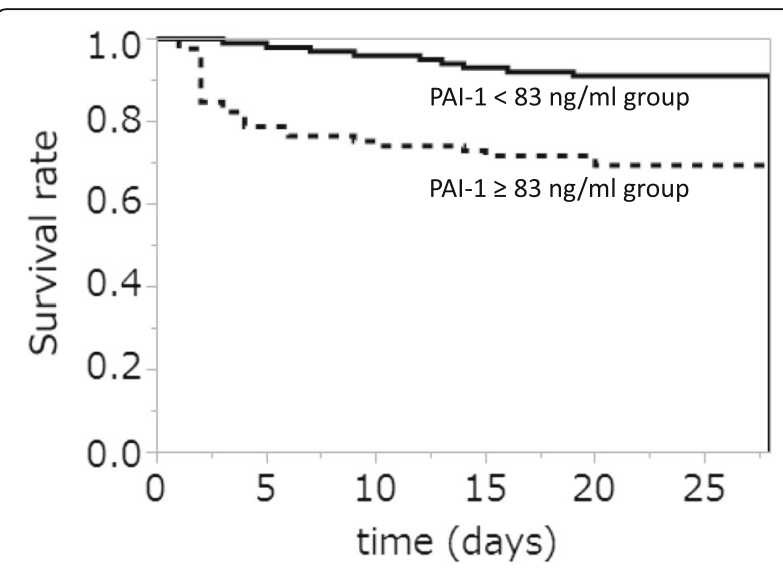

Fig. 2 Kaplan-Meier analysis. The survival rate is significantly higher in the higher group (PAl-1 level $\geq 83 \mathrm{ng} / \mathrm{ml} ; n=85$ ) than that in the lower group (PAl-1 level $<83 \mathrm{ng} / \mathrm{ml} ; n=101)$ (log-rank test, $P<0.01$ )

of $<83 \mathrm{ng} / \mathrm{ml}$ (all $P<0.01$ ). The 28 -day survival rate was significantly lower in patients with a level of $\geq 83 \mathrm{ng} / \mathrm{ml}$ than in those with a PAI-1 level of $<83 \mathrm{ng} / \mathrm{ml}(P<0.01)$. These results suggest that patients with a PAI-1 level of $\geq 83 \mathrm{ng} / \mathrm{ml}$ tend to develop DIC with suppressed fibrinolysis and multiple organ dysfunction. Madoiwa et al. [25] reported that the PAI-1 level correlated with the SOFA score. The results of this previous study are compatible with our results that there was a positive correlation between PAI-1 and the SOFA score in sepsis patients $(r=0.32, P<0.01)$.

Vincent et al. [26] studied the relationship of this dysfunction with the outcome. Increased cardiovascular, central nervous system, or renal SOFA score was related to high mortality. Furthermore, both cardiovascular and renal failures cause the highest mortality. In this study, cardiovascular and renal SOFA scores were significantly higher in the non-survivors' group than in the survivors' group. PAI-1 levels were significantly correlated with cardiovascular and renal scores in each SOFA scores. PAI-1 levels reflect the process that sepsis causes organ failures, resulting in death. Moreover, PAI-1 level is superior to SOFA score for predicting mortality.

Fibrinolysis is suppressed in sepsis and multiple microthrombi impair microcirculation, resulting in organ failure. The PAI-1 level reflects this process and can predict mortality in sepsis. In this study, sepsis was diagnosed according to the new definition of "Sepsis-3." PAI-1 reflects coagulo-fibrinolytic abnormalities, particularly suppressed fibrinolysis and organ failure along with microthrombi, leading to microcirculatory dysfunction in sepsis. Therefore, PAI-1 may be a useful marker to assess sepsis severity according to the Sepsis-3 definition.

The present study has some limitations. This was a small, retrospective, single-center study. The sampling timing in the course of sepsis was not detected. Some 


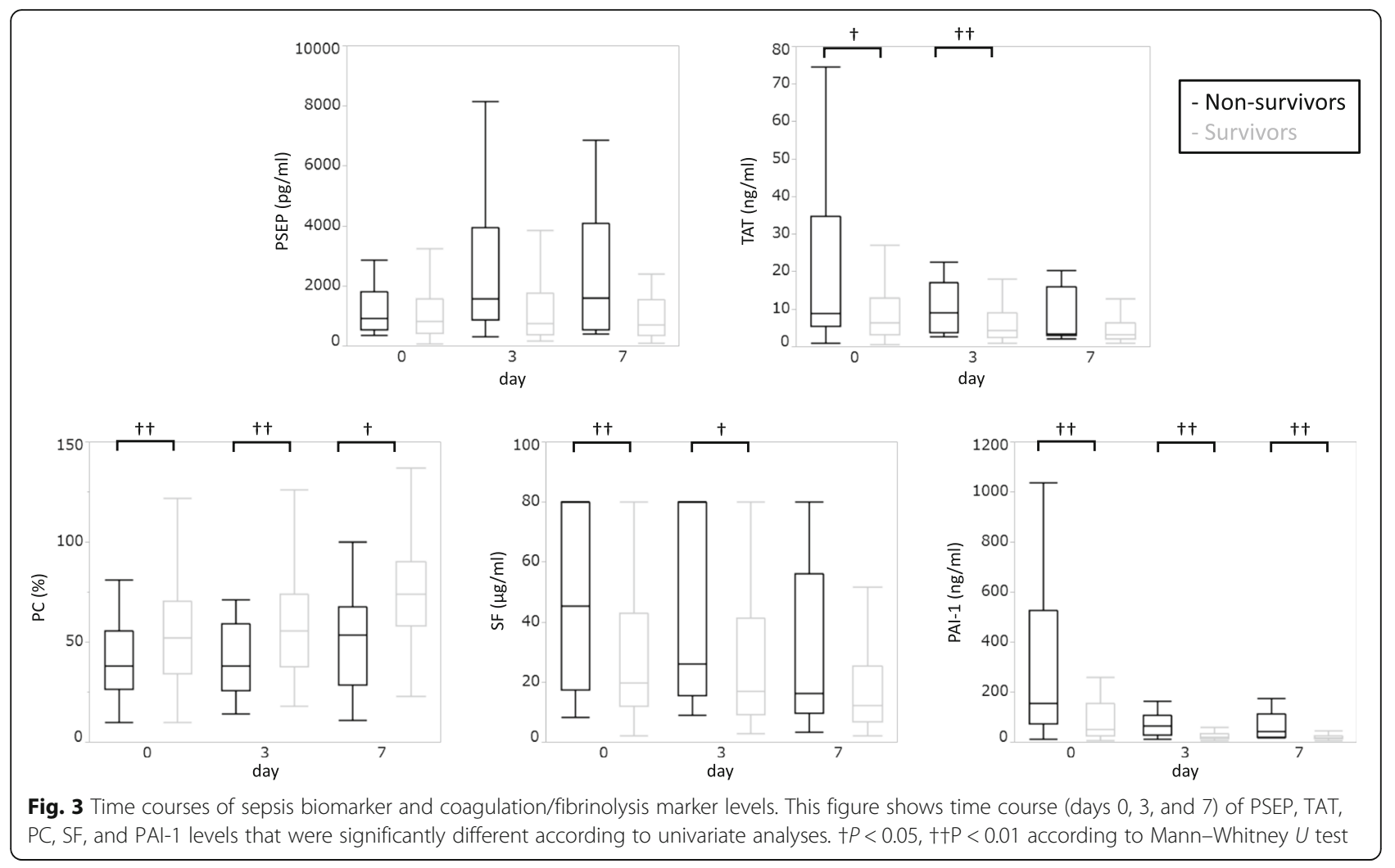

patient characteristics between non-survivors and survivors were different; therefore, further studies are required to make sure of the efficacy of PAI-1. Additionally, only sepsis biomarkers and coagulation/fibrinolysis markers that could be examined in our center were used.

\section{Conclusion}

PAI-1 can predict prognosis in sepsis patients. In addition, PAI-1 reflects DIC with suppressed fibrinolysis and organ failure, with microthrombi leading to microcirculatory dysfunction.

\section{Acknowledgements}

We sincerely thank Ms. Kanae Misumi of the Department of Emergency and Critical Care Medicine, Faculty of Medicine, Fukuoka University for her help in data encoding.

\section{Funding}

We had no external funding sources; each author has funded the research with their own funds.

\section{Availability of data and materials}

The dataset supporting the conclusions of this article is available in data base file in additional materials repository.

\section{Authors' contributions}

$\mathrm{KH}$ drafted the manuscript, participated in the study design, and performed the statistics analysis. TK, YN, YI, NM, YK, and HI helped draft the manuscript. All authors read and approved the final manuscript.
Ethics approval and consent to participate

This study was approved by the institutional review board of Fukuoka University Hospital according to the Declaration of Helsinki. The requirement of informed consent was waived, because this was a retrospective study.

\section{Consent for publication}

Not applicable.

\section{Competing interests}

The authors declare that they have no competing interests.

\section{Publisher's Note}

Springer Nature remains neutral with regard to jurisdictional claims in published maps and institutional affiliations.

Received: 20 March 2017 Accepted: 2 July 2017

Published online: 11 July 2017

\section{References}

1. Vincent JL, Rello J, Marshall J, Silva E, Anzueto A, Martin CD, et al. International study of the prevalence and outcomes of infection in intensive care units. JAMA. 2009;302:2323-9.

2. Vincent JL, Sakr Y, Sprung CL, Ranieri VM, Reinhart K, Gerlach H, et al. Sepsis in European intensive care units: results of the SOAP study. Crit Care Med. 2006;34:344-53.

3. Wittebole X, Castanares-Zapatero D, Laterre PF. Toll-like receptor 4 modulation as a strategy to treat sepsis. Mediators Inflamm. 2010;2010: 568396

4. Endo S, Suzuki Y, Takahashi G, Shozushima T, Ishikura H, Murai A, et al. Usefulness of presepsin in the diagnosis of sepsis in a multicenter prospective study. J Infect Chemother. 2012;18:891-7.

5. Shozushima T, Takahashi G, Matsumoto N, Kojika M, Okamura Y, Endo S. Usefulness of presepsin (SCD14-ST) measurements as a marker for the diagnosis and severity of sepsis that satisfied diagnostic criteria of systemic inflammatory response syndrome. J Infect Chemother. 2011;17:764-9. 
6. Ulla M, Pizzolato E, Lucchiari M, Loiacono M, Soardo F, Forno D, et al. Diagnostic and prognostic value of presepsin in the management of sepsis in the emergency department: a multicenter prospective study. Crit Care. 2013;17:R168.

7. Ito T. PAMPs and DAMPs as triggers for DIC. J Intensive Care. 2014;2:67.

8. Engelmann B, Massberg S. Thrombosis as an intravascular effector of innate immunity. Nat Rev Immunol. 2013;13:34-45.

9. Gando S, Otomo Y. Local hemostasis, immunothrombosis, and systemic disseminated intravascular coagulation in trauma and traumatic shock. Crit Care. 2015;19:72.

10. Rangel-Frausto MS, Pittet D, Costigan M, Hwang T, Davis CS, Wenzel RP. The natural history of the systemic inflammatory response syndrome (SIRS). A prospective study. JAMA. 1995;273:117-23.

11. Dhainaut JF, Yan SB, Joyce DE, Pettilä V, Basson B, Brandt JT, et al. Treatment effects of drotrecogin alfa (activated) in patients with severe sepsis with or without overt disseminated intravascular coagulation. J Thromb Haemost. 2004;2:1924-33.

12. Kienast J, Juers M, Wiedermann CJ, Hoffmann JN, Ostermann H, Strauss R, et al. KyberSept investigators: treatment effects of high-dose antithrombin without concomitant heparin in patients with severe sepsis with or without disseminated intravascular coagulation. J Thromb Haemost. 2006;4:90-7.

13. Beale R, Reinhart K, Brunkhorst FM, Dobb G, Levy M, Martin G, et al. Promoting global research excellence in severe sepsis (PROGRESS): lessons from an international sepsis registry. Infection. 2009;37:222-32.

14. Singer M, Deutschman CS, Seymour CW, Shankar-Hari M, Annane D, Bauer $M$, et al. The Third International Consensus Definitions for Sepsis and Septic Shock (Sepsis-3). JAMA. 2016;315:801-10.

15. Gando S, Iba T, Equchi Y, Ohtomo Y, Okamoto K, Koseki K, et al. A multicenter, prospective validation of disseminated intravascular coagulation diagnostic criteria for critically ill patients: comparing current criteria. Crit Care Med. 2006;34:625-31.

16. Vincent IL, de Mendonça A, Cantraine F, Moreno R, Takala J, Suter PM, et al. Use of the SOFA score to assess the incidence of organ dysfunction/failure in intensive care units: results of a multicenter, prospective study. Working group on "sepsis-related problems" of the European Society of Intensive Care Medicine. Crit Care Med. 1998;26:1793-800.

17. Bone RC, Balk RA, Cerra FB, Dellinger RP, Fein AM, Knaus WA, et al. Definitions for sepsis and organ failure and guidelines for the use of innovative therapies in sepsis. The ACCP/SCCM Consensus Conference Committee. American College of Chest Physicians/Society of Critical Care Medicine Chest. 1992;101:1644-55.

18. Clec'h C, et al. Diagnostic and prognostic value of procalcitonin in patients with septic shock. Crit Care Med. 2004;32:1166-9.

19. Jensen JU, et al. Procalcitonin increase in early identification of critically ill patients at high risk of mortality. Crit Care Med. 2006;34:2596-602.

20. Masson S, et al. Presepsin (soluble CD14 subtype) and procalcitonin levels for mortality in sepsis: data from the Albumin Italian Outcome Sepsis trial. Crit Care. 2014;18:R6.

21. Sprengers ED, Kluft C. Plasminogen activator inhibitors. Blood. 1987;69:381-7.

22. Fujishima S, Gando S, Saitoh D, Mayumi T, Kushimoto S, Shiraishi S, et al. A multicenter, prospective evaluation of quality of care and mortality in Japan based on the Surviving Sepsis Campaign guidelines. J Infect Chemother. 2014;20:115-20

23. Asakura $\mathrm{H}$. Classifying types of disseminated intravascular coagulation: clinical and animal models. J Intensive Care. 2014:2:20.

24. Koyama K, Madoiwa S, Nunomiya S, Koinima T, Wada M, Sakata A, et al. Combination of thrombin-antithrombin complex, plasminogen activator inhibitor-1, and protein C activity for early identification of severe coagulopathy in initial phase of sepsis: a prospective observational study. Crit Care. 2014;18:R13.

25. Madoiwa S, Nunomiya S, Ono T, Shintani Y, Ohmori T, Mimuro J, et al. Plasminogen activator inhibitor 1 promotes a poor prognosis in sepsisinduced disseminated intravascular coagulation. Int J Hematol. 2006;84: 398-405.

26. Vincent JL, Moreno R, Takala J, Willatts S, De Mendonça A, Bruining H, et al. The SOFA (Sepsis-related Organ Failure Assessment) score to describe organ dysfunction/failure. On behalf of the Working Group on Sepsis-Related Problems of the European Society of Intensive Care Medicine. Intensive Care Med. 1996;22(7):707-10

\section{Submit your next manuscript to BioMed Central and we will help you at every step:}

- We accept pre-submission inquiries

- Our selector tool helps you to find the most relevant journal

- We provide round the clock customer support

- Convenient online submission

- Thorough peer review

- Inclusion in PubMed and all major indexing services

- Maximum visibility for your research

Submit your manuscript at www.biomedcentral.com/submit

) Biomed Central 